\title{
Molecular Descriptors of Nanotube, Oxide, Silicate, and Triangulene Networks
}

\author{
Wei Gao ${ }^{1}$ and Muhammad Kamran Siddiqui ${ }^{2}$ \\ ${ }^{1}$ School of Information Science and Technology, Yunnan Normal University, Kunming 650500, China \\ ${ }^{2}$ Department of Mathematics, Comsats Institute of Information Technology, Sahiwal 57000, Pakistan \\ Correspondence should be addressed to Wei Gao; gaowei@ynnu.edu.cn
}

Received 5 September 2016; Accepted 4 December 2016; Published 5 February 2017

Academic Editor: Teik-Cheng Lim

Copyright (C) 2017 Wei Gao and Muhammad Kamran Siddiqui. This is an open access article distributed under the Creative Commons Attribution License, which permits unrestricted use, distribution, and reproduction in any medium, provided the original work is properly cited.

\begin{abstract}
A topological index is a real number associated with chemical constitution purporting for correlation of chemical structure with various physical properties, chemical reactivity, or biological activity. The concept of hyper Zagreb index, first multiple Zagreb index, second multiple Zagreb index, and Zagreb polynomials was established in chemical graph theory based on vertex degrees. It is reported that these indices are useful in the study of anti-inflammatory activities of certain chemical networks. In this paper, we study carbon nanotube networks which are motivated by molecular structure of regular hexagonal lattice and also studied interconnection networks which are motivated by molecular structure of a chemical compound $\mathrm{SiO}_{4}$. We determine hyper Zagreb index, first multiple Zagreb index, second multiple Zagreb index, and Zagreb polynomials for some important class of carbon nanotube networks, dominating oxide network, dominating silicate network, and regular triangulene oxide network.
\end{abstract}

\section{Introduction}

Multiprocessorinterconnection networks are regularly required to associate a huge number of homogeneously repeated processor-memory matches, each of which is known as a preparing hub. Rather than utilizing a mutual memory, all synchronization and communication between processing nodes for program execution is often done via message passing. Outline and utilization of multiprocessor interconnection systems have as of late attracted extensive consideration due to the accessibility of modest, capable microchips, and memory chips. The work systems have been perceived as adaptable interconnection systems for massively parallel computing; see [1]. Work/torus-like low-dimensional systems have as of late gotten a considerable measure of consideration for their better adaptability to bigger systems, rather than more mind boggling systems, for example, hypercubes. There is a lot of relevant works on interdependent networks which can be reviewed. In particular the failure of cooperation on dependent networks has been studied a lot recently in [2-5].
Particles and subnuclear blends are as often as possible showed by subnuclear graphs. A subnuclear outline is a graph in which vertices are particles of a given molecule and edges are its compound bonds. Since the valency of carbon is four, it is customary to consider all graphs with most outrageous degree under 4 , as a molecular outline. An outline $G(V, E)$ with vertex set $V$ and edge set $E$ is related, if there exists a connection between any match of vertices in $G$. $A$ framework is just a connected graph having no various edges and no circles. All through in this article, the level of vertex $v \in V(G)$ is implied by $\operatorname{deg}(v)$ and is the amount of edges incident to $v$.

A topological index is a numeric amount connected with a graph which describes the topology of diagram and is invariant under chart automorphism. In more exact way, a topological list $\operatorname{Top}(G)$ of graph $G$ is a number with the property that, for each diagram $H$ isomorphic to chart $G$, $\operatorname{Top}(H)=\operatorname{Top}(G)$. The idea of topological list originated from work done by Wiener [6] while he was chipping away at breaking point of paraffin. He named this list as way number. Later on, the way number was renamed as Wiener index. The 
Wiener file is the first and the most concentrated topological file, both from hypothetical perspective and applications, and is characterized as the entirety of separation between all sets of vertices in $G$; see for details $[7,8]$.

One of the oldest topological indices is the first Zagreb index introduced by Gutman and Trinajstić based on degree of vertices of $G$ in 1972 [4]. The first and second Zagreb indices of a graph $G$ are defined as

$$
\begin{aligned}
& M_{1}(G)=\sum_{u v \in E(G)}[\operatorname{deg}(u)+\operatorname{deg}(v)], \\
& M_{2}(G)=\sum_{u v \in E(G)}[\operatorname{deg}(u) \times \operatorname{deg}(v)] .
\end{aligned}
$$

In 2013, Shirdel et al. [9] introduced a new degree based of Zagreb index named "hyper Zagreb index" as

$$
H M(G)=\sum_{u v \in E(G)}[\operatorname{deg}(u)+\operatorname{deg}(v)]^{2} .
$$

Ghorbani and Azimi defined two new versions of Zagreb indices of a graph $G$ in 2012 [2]. These indices are the first multiple Zagreb index $P M_{1}(G)$ and the second multiple Zagreb index $\mathrm{PM}_{2}(G)$ and these indices are defined as follows:

$$
\begin{aligned}
& P M_{1}(G)=\prod_{u v \in E(G)}[\operatorname{deg}(u)+\operatorname{deg}(v)], \\
& P M_{2}(G)=\prod_{u v \in E(G)}[\operatorname{deg}(u) \times \operatorname{deg}(v)] .
\end{aligned}
$$

The properties of $P M_{1}(G)$ and $P M_{2}(G)$ indices for some chemical structures have been studied in $[2,10]$.

The first Zagreb polynomial $M_{1}(G, x)$ and the second Zagreb polynomial $M_{2}(G, x)$ are defined as follows:

$$
\begin{aligned}
& M_{1}(G, x)=\sum_{u v \in E(G)} x^{[\operatorname{deg}(u)+\operatorname{deg}(v)]}, \\
& M_{2}(G, x)=\sum_{u v \in E(G)} x^{[\operatorname{deg}(u) \times \operatorname{deg}(v)]} .
\end{aligned}
$$

The properties of $M_{1}(G, x)$ and $M_{2}(G, x)$ polynomials for some chemical structures have been studied in [3].

Nowadays there is an extensive research activity on $H M(G), P M_{1}(G)$, and $P M_{2}(G)$ indices and $M_{1}(G, x)$ and $M_{2}(G, x)$ polynomials and their variants; see also [2, 4, 9-14].

For further study of topological indices of various graph families, see $[8,11,12,14-27]$.

\section{Carbon Nanotube Networks}

The disclosure of the fullerene particles and related types of carbon structures, for example, nanotubes, has created an explosion of movement in science, physical science, and materials science. Traditional fullerene is an all-carbon particle in which the iotas are masterminded on a pseudospherical structure made up altogether of pentagons and hexagons. Its subatomic diagram is a finite trivalent chart installed on the

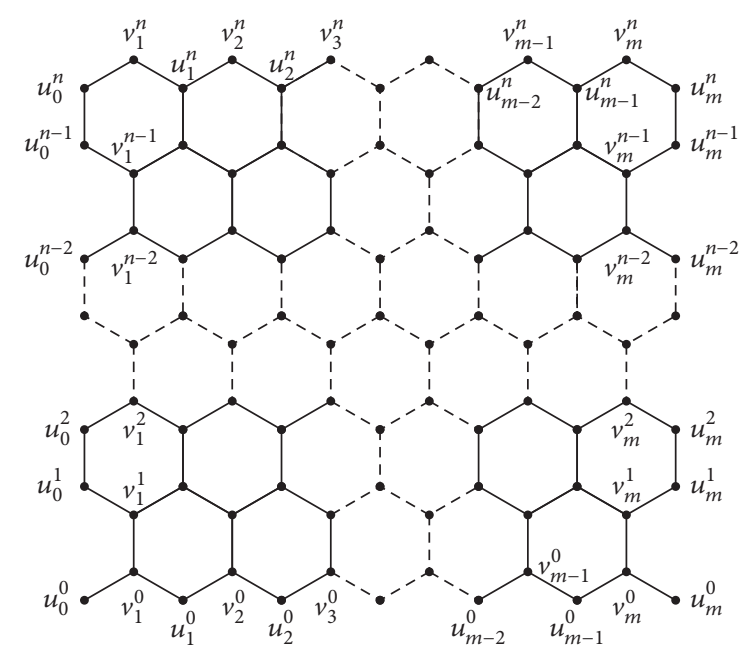

FIgURE 1: Quadrilateral section $P_{m}^{n}$ cuts from the regular hexagonal lattice.

surface of a sphere with just hexagonal and (precisely 12) pentagonal appearances. Deza et al. [28] considered fullerene's augmentation to other shut surfaces and demonstrated that exclusive four surfaces are conceivable, in particular, circle, torus, Klein bottle, and projective plane. Not at all like circular fullerenes, toroidal and Klein container's fullerenes have been viewed as decorations of whole hexagons on their surfaces since they should contain no pentagons; see [17, 18, 28-33].

Carbon nanotubes are allotropes of carbon with a cylindrical nanostructure. These round and hollow carbon atoms have abnormal properties, which are significant for nanotechnology, hardware, optics, and different fields of materials science and innovation. Nanotubes are individuals from the fullerene auxiliary family. Their name is gotten from their long, empty structure.

Let us consider the $m \times n$ quadrilateral section $P_{m}^{n}$ with $m \geq 2$ hexagons on the top and bottom sides and $n \geq 2$ hexagons on the lateral sides cut from the regular hexagonal lattice $L$; see Figure 1 .

If we identify two lateral sides of $P_{m}^{n}$ such that we identify vertices $u_{0}^{j}$ and $u_{m}^{j}$, for $j=0,1,2, \ldots, n$, then we obtain nanotube $\mathbb{N A}_{m}^{n}$ with $2 m(n+1)$ vertices and $(3 n+2) m$ edges.

Let $n$ be even, $n \geq 2$, and $m \geq 2$. If we identify the top and bottom sides of the quadrilateral section $P_{m}^{n}$ in such a way that we identify vertices $u_{i}^{0}$ and $u_{i}^{n}$, for $i=0,1,2, \ldots, m$, and vertices $v_{i}^{0}$ and $v_{i}^{n}$, for $i=1,2, \ldots, m$, then we obtain nanotube $\mathbb{N C}_{m}^{n}$ of order $n(2 m+1)$ and size $(3 m n+n / 2)$.

Recently some well known topological indices, namely, Randić index, atom-bond connectivity index, and geometric arithmetic index for nanotube $\mathbb{N} A_{m}^{n}$ and nanotube $\mathbb{N C}_{m}^{n}$ have been studied in $[17,18]$.

Now we compute hyper Zagreb index $H M(G)$, first multiple Zagreb index $P M_{1}(G)$, second multiple Zagreb index $P M_{2}(G)$, and Zagreb polynomials $M_{1}(G, x)$ and $M_{2}(G, x)$ for nanotube $\mathbb{N} A_{m}^{n}$. 
Theorem 1. Consider nanotube $\mathbb{N A}_{m}^{n}$ for $m, n \geq 2$. Then

$$
\begin{aligned}
H M\left(\mathbb{N}_{m}^{n}\right) & =108 m n+28 m, \\
P M_{1}\left(\mathbb{N A}_{m}^{n}\right) & =6^{3 m n-2 m} \times 5^{4 m}, \\
P M_{2}\left(\mathbb{N A}_{m}^{n}\right) & =9^{3 m n-2 m} \times 6^{4 m}, \\
M_{1}\left(\mathbb{N} A_{m}^{n}, x\right) & =(3 m n-2 m) x^{6}+4 m x^{5}, \\
M_{2}\left(\mathbb{N A}_{m}^{n}, x\right) & =(3 m n-2 m) x^{9}+4 m x^{6} .
\end{aligned}
$$

Proof. Nanotube $\mathbb{N} A_{m}^{n}$ has $2 m$ vertices of degree 2 and $2 m n$ vertices of degree 3 . The edge set $E\left(\mathbb{N} \mathbb{A}_{m}^{n}\right)$ divides into two edge partitions based on degrees of end vertices. The first edge partition $E_{1}\left(\mathbb{N}_{m}^{n}\right)$ contains $m(3 n-2)$ edges $u v$, where $\operatorname{deg}(u)=\operatorname{deg}(v)=3$. The second edge partition $E_{2}\left(\mathbb{N A}_{m}^{n}\right)$ contains $4 m$ edges $u v$, where $\operatorname{deg}(u)=2$ and $\operatorname{deg}(v)=3$. Now using (2)-(4), we have

$$
\begin{aligned}
& H M(G)=\sum_{u v \in E(G)}[\operatorname{deg}(u)+\operatorname{deg}(v)]^{2} \\
& H M\left(\mathbb{N A}_{m}^{n}\right)=\sum_{u v \in E_{1}\left(\mathbb{N A}_{m}^{n}\right)}[\operatorname{deg}(u)+\operatorname{deg}(v)]^{2} \\
& +\sum_{u v \in E_{2}\left(\mathbb{N A}_{m}^{n}\right)}[\operatorname{deg}(u)+\operatorname{deg}(v)]^{2} \\
& =6^{2}\left|E_{1}\left(\mathbb{N} \mathbb{A}_{m}^{n}\right)\right|+5^{2}\left|E_{2}\left(\mathbb{N} \mathbb{A}_{m}^{n}\right)\right| \\
& =36(3 m n-2 m)+25(4 m) \\
& =108 m n+28 m \text {, } \\
& P M_{1}(G)=\prod_{u v \in E(G)}[\operatorname{deg}(u)+\operatorname{deg}(v)] \\
& P M_{1}\left(\mathbb{N} \mathbb{A}_{m}^{n}\right)=\prod_{u v \in E_{1}\left(\mathbb{N A}_{m}^{n}\right)}[\operatorname{deg}(u)+\operatorname{deg}(v)] \\
& \times \prod_{u v \in E_{2}\left(\mathbb{N A}_{m}^{n}\right)}[\operatorname{deg}(u)+\operatorname{deg}(v)] \\
& =6^{\left|E_{1}\left(\mathbb{N} A_{m}^{n}\right)\right|} \times 5^{\left|E_{2}\left(\mathbb{N} A_{m}^{n}\right)\right|} \\
& =6^{(3 m n-2 m)} \times 5^{4 m}, \\
& P M_{2}(G)=\prod_{u v \in E(G)}[\operatorname{deg}(u) \times \operatorname{deg}(v)], \\
& P M_{2}\left(\mathbb{N} A_{m}^{n}\right)=\prod_{u v \in E_{1}\left(\mathbb{N A}_{m}^{n}\right)}[\operatorname{deg}(u) \times \operatorname{deg}(v)] \\
& \times \prod_{u v \in E_{2}\left(\mathbb{N A}_{m}^{n}\right)}[\operatorname{deg}(u) \times \operatorname{deg}(v)] \\
& =9^{\left|E_{1}\left(\mathbb{N} A_{m}^{n}\right)\right|} \times 6^{\left|E_{2}\left(\mathbb{N} A_{m}^{n}\right)\right|} \\
& =9^{(3 m n-2 m)} \times 6^{4 m},
\end{aligned}
$$

$$
\begin{aligned}
& M_{1}(G, x)=\sum_{u v \in E(G)} x^{[\operatorname{deg}(u)+\operatorname{deg}(v)]}, \\
& M_{1}\left(\mathbb{N}_{m}^{n}, x\right)=\sum_{u v \in E_{1}\left(\mathbb{N A}_{m}^{n}\right)} x^{[\operatorname{deg}(u)+\operatorname{deg}(v)]} \\
& +\sum_{u v \in E_{2}\left(\mathbb{N A}_{m}^{n}\right)} x^{[\operatorname{deg}(u)+\operatorname{deg}(v)]} \\
& =\sum_{u v \in E_{1}\left(\mathbb{N A}_{m}^{n}\right)} x^{6}+\sum_{u v \in E_{2}\left(\mathbb{N A}_{m}^{n}\right)} x^{5} \\
& =\left|E_{1}\left(\mathbb{N} A_{m}^{n}\right)\right| x^{6}+\left|E_{2}\left(\mathbb{N} A_{m}^{n}\right)\right| x^{5} \\
& =(3 m n-2 m) x^{6}+4 m x^{5} \text {, } \\
& M_{2}(G, x)=\sum_{u v \in E(G)} x^{[\operatorname{deg}(u) \times \operatorname{deg}(v)]}, \\
& M_{2}\left(\mathbb{N} A_{m}^{n}, x\right)=\sum_{u v \in E_{1}\left(\mathbb{N A}_{m}^{n}\right)} x^{[\operatorname{deg}(u) \times \operatorname{deg}(v)]} \\
& +\sum_{u v \in E_{2}\left(\mathbb{N A}_{m}^{n}\right)} x^{[\operatorname{deg}(u) \times \operatorname{deg}(v)]} \\
& =\sum_{u v \in E_{1}\left(\mathbb{N A}_{m}^{n}\right)} x^{9}+\sum_{u v \in E_{2}\left(\mathbb{N A}_{m}^{n}\right)} x^{6} \\
& =\left|E_{1}\left(\mathbb{N} \mathbb{A}_{m}^{n}\right)\right| x^{9}+\left|E_{2}\left(\mathbb{N} \mathbb{A}_{m}^{n}\right)\right| x^{6} \\
& =(3 m n-2 m) x^{9}+4 m x^{6} \text {. }
\end{aligned}
$$

Now we compute hyper Zagreb index $H M(G)$, first multiple Zagreb index $P M_{1}(G)$, second multiple Zagreb index $P M_{2}(G)$, and Zagreb polynomials $M_{1}(G, x)$ and $M_{2}(G, x)$ for nanotube $\mathbb{N C}_{m}^{n}$.

Theorem 2. Consider nanotube $\mathbb{N C}_{m}^{n}$ for $n \geq 2$ even and $m \geq$ 2. Then

$$
\begin{aligned}
& H M\left(\mathbb{N C}_{m}^{n}\right)=108 m n-24 n \\
& P M_{1}\left(\mathbb{N C}_{m}^{n}\right)=4^{n} \times 5^{2 n} \times 6^{(6 m n-5 n) / 2}, \\
& P M_{2}\left(\mathbb{N C}_{m}^{n}\right)=4^{n} \times 6^{2 n} \times 9^{(6 m n-5 n) / 2}, \\
& M_{1}\left(\mathbb{N C}_{m}^{n}, x\right)=n x^{4}+2 n x^{5}+\left(\frac{6 m n-5 n}{2}\right) x^{6}, \\
& M_{2}\left(\mathbb{N C}_{m}^{n}, x\right)=n x^{4}+2 n x^{6}+\left(\frac{6 m n-5 n}{2}\right) x^{9}
\end{aligned}
$$

Proof. Nanotube $\mathbb{N C}_{m}^{n}$ has $2 n$ vertices of degree 2 and $n(2 m-$ $1)$ vertices of degree 3 . There are three types of edges in $E\left(\mathbb{N C}_{m}^{n}\right)$ based on degrees of end vertices of each edge; that is, $E\left(\mathbb{N C}_{m}^{n}\right)=E_{1}\left(\mathbb{N C}_{m}^{n}\right) \cup E_{2}\left(\mathbb{N C}_{m}^{n}\right) \cup E_{3}\left(\mathbb{N C}_{m}^{n}\right)$. Edge partition $E_{1}\left(\mathbb{N C}_{m}^{n}\right)$ contains $n$ edges $u v$, where $\operatorname{deg}(u)=\operatorname{deg}(v)=2$; edge partition $E_{2}\left(\mathbb{N C}_{m}^{n}\right)$ contains $2 n$ edges $u v$, where $\operatorname{deg}(u)=$ 2 and $\operatorname{deg}(v)=3$, and edge partition $E_{3}\left(\mathbb{N C}_{m}^{n}\right)$ contains 
$(6 m n-5 n) / 2$ edges $u v$, where $\operatorname{deg}(u)=\operatorname{deg}(v)=3$. Now using (2)-(4), we have

$$
\begin{aligned}
& H M(G)=\sum_{u v \in E(G)}[\operatorname{deg}(u)+\operatorname{deg}(v)]^{2}, \\
& H M\left(\mathbb{N C}_{m}^{n}\right)=\sum_{u v \in E_{1}\left(\mathbb{N C}_{m}^{n}\right)}[\operatorname{deg}(u)+\operatorname{deg}(v)]^{2} \\
& +\sum_{u v \in E_{2}\left(\mathbb{N C}_{m}^{n}\right)}[\operatorname{deg}(u)+\operatorname{deg}(v)]^{2} \\
& +\sum_{u v \in E_{3}\left(\mathbb{N C}_{m}^{n}\right)}[\operatorname{deg}(u)+\operatorname{deg}(v)]^{2} \\
& =4^{2}\left|E_{1}\left(\mathbb{N C}_{m}^{n}\right)\right|+5^{2}\left|E_{2}\left(\mathbb{N C}_{m}^{n}\right)\right| \\
& +6^{2}\left|E_{3}\left(\mathbb{N C}_{m}^{n}\right)\right| \\
& =16 n+25(2 n)+36\left(\frac{6 m n-5 n}{2}\right) \\
& =108 m n-24 n, \\
& P M_{1}(G)=\prod_{u v \in E(G)}[\operatorname{deg}(u)+\operatorname{deg}(v)], \\
& P M_{1}\left(\mathbb{N C}_{m}^{n}\right)=\prod_{u v \in E_{1}\left(\mathbb{N C}_{m}^{n}\right)}[\operatorname{deg}(u)+\operatorname{deg}(v)] \\
& \times \prod_{u v \in E_{2}\left(\mathbb{N C}_{m}^{n}\right)}[\operatorname{deg}(u)+\operatorname{deg}(v)] \\
& \times \prod_{u v \in E_{3}\left(\mathbb{N C}_{m}^{n}\right)}[\operatorname{deg}(u)+\operatorname{deg}(v)] \\
& =4^{\left|E_{1}\left(\mathbb{N C}_{m}^{n}\right)\right|} \times 5^{\left|E_{2}\left(\mathbb{N} C_{m}^{n}\right)\right|} \times 6^{\left|E_{3}\left(\mathbb{N} C_{m}^{n}\right)\right|} \\
& =4^{n} \times 5^{2 n} \times 6^{(6 m n-5 n) / 2} \text {, } \\
& P_{2}(G)=\prod_{u v \in E(G)}[\operatorname{deg}(u) \times \operatorname{deg}(v)], \\
& P M_{2}\left(\mathbb{N C}_{m}^{n}\right)=\prod_{u v \in E_{1}\left(\mathbb{N C}_{m}^{n}\right)}[\operatorname{deg}(u) \times \operatorname{deg}(v)] \\
& \times \prod_{u v \in E_{2}\left(\mathbb{N C}_{m}^{n}\right)}[\operatorname{deg}(u) \times \operatorname{deg}(v)] \\
& \times \prod_{u v \in E_{3}\left(\mathbb{N C}_{m}^{n}\right)}[\operatorname{deg}(u) \times \operatorname{deg}(v)] \\
& =4^{\left|E_{1}\left(\mathbb{N} C_{m}^{n}\right)\right|} \times 6^{\left|E_{2}\left(\mathbb{N C}_{m}^{n}\right)\right|} \times 9^{\left|E_{3}\left(\mathbb{N C}_{m}^{n}\right)\right|} \\
& =4^{n} \times 6^{2 n} \times 9^{(6 m n-5 n) / 2} \text {, } \\
& M_{1}(G, x)=\sum_{u v \in E(G)} x^{[\operatorname{deg}(u)+\operatorname{deg}(v)]}, \\
& M_{1}\left(\mathbb{N C}_{m}^{n}, x\right)=\sum_{u v \in E_{1}\left(\mathbb{N C}_{\mathrm{m}}^{n}\right)} x^{[\operatorname{deg}(u)+\operatorname{deg}(v)]} \\
& +\sum_{u v \in E_{2}\left(\mathbb{N C}_{m}^{n}\right)} x^{[\operatorname{deg}(u)+\operatorname{deg}(v)]}
\end{aligned}
$$

$$
\begin{aligned}
& +\sum_{u v \in E_{3}\left(\mathbb{N C}_{m}^{n}\right)} x^{[\operatorname{deg}(u)+\operatorname{deg}(v)]} \\
& =\sum_{u v \in E_{1}\left(\mathbb{N C}_{m}^{n}\right)} x^{4}+\sum_{u v \in E_{2}\left(\mathbb{N} C_{m}^{n}\right)} x^{5} \\
& +\sum_{u v \in E_{3}\left(\mathbb{N C}_{m}^{n}\right)} x^{6} \\
& =\left|E_{1}\left(\mathbb{N C}_{m}^{n}\right)\right| x^{4}+\left|E_{2}\left(\mathbb{N C}_{m}^{n}\right)\right| x^{5} \\
& +\left|E_{3}\left(\mathbb{N C}_{m}^{n}\right)\right| x^{6} \\
& =n x^{4}+2 n x^{5}+\left(\frac{6 m n-5 n}{2}\right) x^{6}, \\
& M_{2}(G, x)=\sum_{u v \in E(G)} x^{[\operatorname{deg}(u) \times \operatorname{deg}(v)]}, \\
& M_{2}\left(\mathbb{N C}_{m}^{n}, x\right)=\sum_{u v \in E_{1}\left(\mathbb{N C}_{m}^{n}\right)} x^{[\operatorname{deg}(u) \times \operatorname{deg}(v)]} \\
& +\sum_{u v \in E_{2}\left(\mathbb{N C}_{m}^{n}\right)} x^{[\operatorname{deg}(u) \times \operatorname{deg}(v)]} \\
& +\sum_{u v \in E_{3}\left(\mathbb{N C}_{m}^{n}\right)} x^{[\operatorname{deg}(u) \times \operatorname{deg}(v)]} \\
& =\sum_{u v \in E_{1}\left(\mathbb{N C}_{m}^{n}\right)} x^{4}+\sum_{u v \in E_{2}\left(\mathbb{N C}_{m}^{n}\right)} x^{6} \\
& +\sum_{u v \in E_{3}\left(\mathbb{N C}_{m}^{n}\right)} x^{9} \\
& =\left|E_{1}\left(\mathbb{N C}_{m}^{n}\right)\right| x^{4}+\left|E_{2}\left(\mathbb{N C}_{m}^{n}\right)\right| x^{6} \\
& +\left|E_{3}\left(\mathbb{N C}_{m}^{n}\right)\right| x^{9} \\
& =n x^{4}+2 n x^{6}+\left(\frac{6 m n-5 n}{2}\right) x^{9} \text {. }
\end{aligned}
$$

\section{Dominating Oxide Network DOX $(n)$}

In 2015, Baig et al. [34] computed general Randić index, atom-bond connectivity $(\mathrm{ABC})$ index, and geometric arithmetic (GA) index of dominating oxide network $\operatorname{DOX}(n)$. In this section, we calculate hyper Zagreb index $H M(G)$, first multiple Zagreb index $P M_{1}(G)$, second multiple Zagreb index $P M_{2}(G)$, and Zagreb polynomials $M_{1}(G, x)$ and $M_{2}(G, x)$ for dominating oxide network $\operatorname{DOX}(n)$.

Theorem 3. Consider the dominating oxide network $\operatorname{DOX}(n)$ for $n \geq 2$. Then

$$
\begin{aligned}
& H M(D O X(n))=3456 n^{2}-4128 n+1488, \\
& P M_{1}(\operatorname{DOX}(n))=6^{(24 n-12)} \times 8^{\left(54 n^{2}-78 n+30\right)}, \\
& P M_{2}(\operatorname{DOX}(n))=8^{(24 n-12)} \times 16^{\left(54 n^{2}-78 n+30\right)},
\end{aligned}
$$




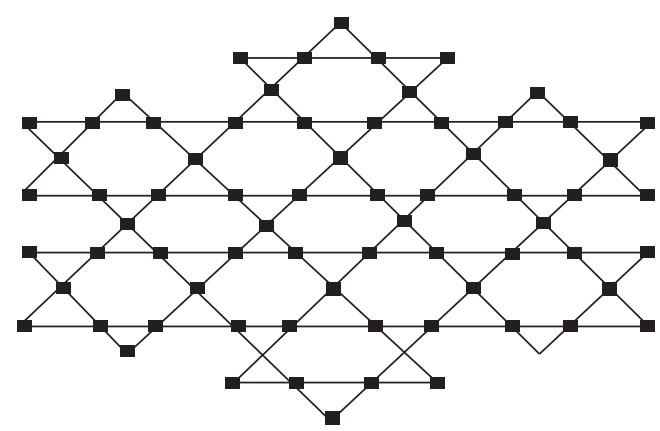

FIgURE 2: Dominating oxide network (DOX(2)).

$$
\begin{aligned}
M_{1}(\operatorname{DOX}(n), x)= & (24 n-12) x^{6} \\
& +\left(54 n^{2}-78 n+30\right) x^{8}, \\
M_{2}(\operatorname{DOX}(n), x)= & (24 n-12) x^{8} \\
& +\left(54 n^{2}-78 n+30\right) x^{16} .
\end{aligned}
$$

Proof. Let $\operatorname{DOX}(n)$ be the dominating oxide network. The number of vertices and edges in $\operatorname{DOX}(n)$ are $27 n^{2}-21 n+6$ and $54 n^{2}-54 n+18$, respectively; see Figure 2 . The edge set $E(\operatorname{DOX}(n))$ divides into two edge partitions based on degrees of end vertices. The first edge partition $E_{1}(\operatorname{DOX}(n))$ contains $24 n-12$ edges $u v$, where $\operatorname{deg}(u)=2, \operatorname{deg}(v)=4$. The second edge partition $E_{2}(\operatorname{DOX}(n))$ contains $54 n^{2}-78 n+30$ edges $u v$, where $\operatorname{deg}(u)=\operatorname{deg}(v)=4$.

Now using (2)-(4), we have

$$
H M(G)=\sum_{u v \in E(G)}[\operatorname{deg}(u)+\operatorname{deg}(v)]^{2},
$$

$$
H M(\operatorname{DOX}(n))
$$

$$
\begin{gathered}
=\sum_{u v \in E_{1}(\operatorname{DOX}(n))}[\operatorname{deg}(u)+\operatorname{deg}(v)]^{2} \\
+\sum_{u v \in E_{2}(\operatorname{DOX}(n))}[\operatorname{deg}(u)+\operatorname{deg}(v)]^{2} \\
=6^{2}\left|E_{1}(\operatorname{DOX}(n))\right|+8^{2}\left|E_{2}(\operatorname{DOX}(n))\right| \\
=3456 n^{2}-4128 n+1488, \\
P M_{1}(G)=\prod_{u v \in E(G)}[\operatorname{deg}(u)+\operatorname{deg}(v)], \\
P M_{1}(\operatorname{DOX}(n)) \\
=\prod_{u v \in E_{1}(\operatorname{DOX}(n))}[\operatorname{deg}(u)+\operatorname{deg}(v)] \\
\quad \times \prod_{u v \in E_{2}(\operatorname{DOX}(n))}[\operatorname{deg}(u)+\operatorname{deg}(v)]
\end{gathered}
$$

$$
\begin{aligned}
& =6^{\left|E_{1}(\operatorname{DOX}(n))\right|} \times 8^{\left|E_{2}(\operatorname{DOX}(n))\right|} \\
& =6^{(24 n-12)} \times 8^{\left(54 n^{2}-78 n+30\right)} \text {, } \\
& P M_{2}(G)=\prod_{u v \in E(G)}[\operatorname{deg}(u) \times \operatorname{deg}(v)], \\
& P M_{2}(\operatorname{DOX}(n)) \\
& =\prod_{u v \in E_{1}(\operatorname{DOX}(n))}[\operatorname{deg}(u) \times \operatorname{deg}(v)] \\
& \times \prod_{u v \in E_{2}(\operatorname{DOX}(n))}[\operatorname{deg}(u) \times \operatorname{deg}(v)] \\
& =8^{\left|E_{1}(\operatorname{DOX}(n))\right|} \times 16^{\left|E_{2}(\operatorname{DOX}(n))\right|} \\
& =8^{(24 n-12)} \times 16^{\left(54 n^{2}-78 n+30\right)}, \\
& M_{1}(G, x)=\sum_{u v \in E(G)} x^{[\operatorname{deg}(u)+\operatorname{deg}(v)]}, \\
& M_{1}(\operatorname{DOX}(n), x) \\
& =\sum_{u v \in E_{1}(\operatorname{DOX}(n))} x^{[\operatorname{deg}(u)+\operatorname{deg}(v)]} \\
& +\sum_{u v \in E_{2}(\operatorname{DOX}(n))} x^{[\operatorname{deg}(u)+\operatorname{deg}(v)]} \\
& =\sum_{u v \in E_{1}(\operatorname{DOX}(n))} x^{6}+\sum_{u v \in E_{2}(\operatorname{DOX}(n))} x^{8} \\
& =\left|E_{1}(\operatorname{DOX}(n))\right| x^{6}+\left|E_{2}(\operatorname{DOX}(n))\right| x^{8} \\
& =(24 n-12) x^{6}+\left(54 n^{2}-78 n+30\right) x^{8}, \\
& M_{2}(G, x)=\sum_{u v \in E(G)} x^{[\operatorname{deg}(u) \times \operatorname{deg}(v)]} \\
& M_{2}(\operatorname{DOX}(n), x) \\
& =\sum_{u v \in E_{1}(\operatorname{DOX}(n))} x^{[\operatorname{deg}(u) \times \operatorname{deg}(v)]} \\
& +\sum_{u v \in E_{2}(\operatorname{DOX}(n))} x^{[\operatorname{deg}(u) \times \operatorname{deg}(v)]} \\
& =\sum_{u v \in E_{1}(\operatorname{DOX}(n))} x^{8}+\sum_{u v \in E_{2}(\operatorname{DOX}(n))} x^{16} \\
& =\left|E_{1}(\operatorname{DOX}(n))\right| x^{8}+\left|E_{2}(\operatorname{DOX}(n))\right| x^{16} \\
& =(24 n-12) x^{8}+\left(54 n^{2}-78 n+30\right) x^{16} \text {. }
\end{aligned}
$$

\section{Dominating Silicate Network DSL $(n)$}

In 2015, Baig et al. [34] computed general Randić index, atom-bond connectivity $(\mathrm{ABC})$ index, and geometric arithmetic (GA) index of dominating silicate network $\operatorname{DSL}(n)$. 


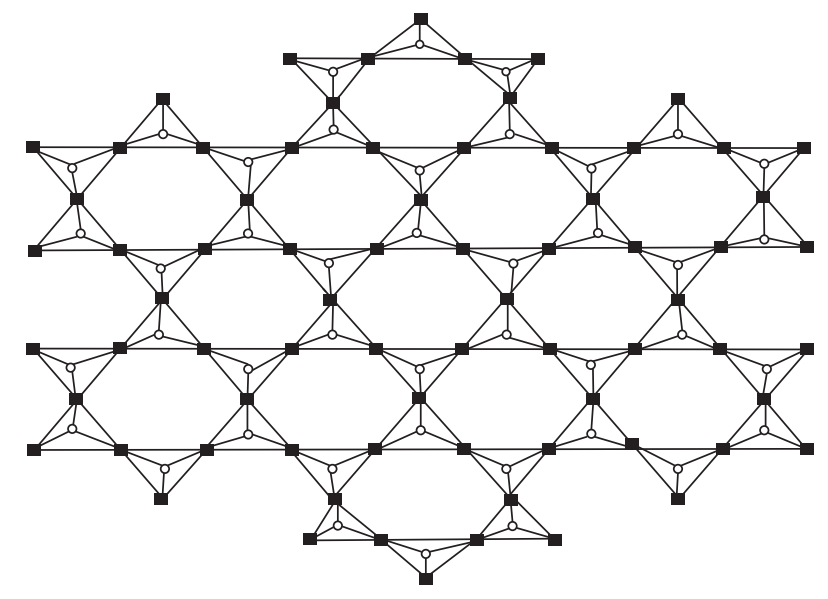

FIgURE 3: Dominating silicate network (DSL(2)).

In this section, we calculate hyper Zagreb index $H M(G)$, first multiple Zagreb index $P M_{1}(G)$, second multiple Zagreb index $P M_{2}(G)$, and Zagreb polynomials $M_{1}(G, x)$ and $M_{2}(G, x)$ for dominating silicate network $\operatorname{DSL}(n)$.

Theorem 4. Consider the dominating silicate network DSL(n) for $n \geq 2$. Then

$$
\begin{aligned}
H M(D S L(n))= & 11070 n^{2}-13452 n+4746 \\
P M_{1}(D S L(n))=5^{(12 n-6)} \times 8^{(24 n-12)} \times 9^{\left(54 n^{2}-66 n+24\right)} & \times 12^{\left(54 n^{2}-78 n+30\right)} \\
P M_{2}(D S L(n))=6^{(12 n-6)} \times 12^{(24 n-12)} & \times 18^{\left(54 n^{2}-66 n+24\right)} \\
& \times 36^{\left(54 n^{2}-78 n+30\right)} \\
M_{1}(D S L(n), x)= & (12 n-6) x^{5}+(24 n-12) x^{8} \\
& +\left(54 n^{2}-66 n+24\right) x^{9} \\
& +\left(54 n^{2}-78 n+30\right) x^{12} \\
M_{2}(D S L(n), x)= & (12 n-6) x^{6}+(24 n-12) x^{12} \\
& +\left(54 n^{2}-66 n+24\right) x^{18} \\
& +\left(54 n^{2}-78 n+30\right) x^{36}
\end{aligned}
$$

Proof. Let $\operatorname{DSL}(n)$ be the dominating silicate network; see Figure 3. The number of vertices and edges in $\operatorname{DSL}(n)$ is $45 n^{2}-39 n+12$ and $108 n^{2}-108 n+36$, respectively. The edge set $E(\operatorname{DSL}(n))$ divides into four edge partitions based on degrees of end vertices. The first edge partition $E_{1}(\operatorname{DSL}(n))$ contains $12 n-6$ edges $u v$, where $\operatorname{deg}(u)=2, \operatorname{deg}(v)=3$. The second edge partition $E_{2}(\operatorname{DSL}(n))$ contains $24 n-12$ edges $u v$, where $\operatorname{deg}(u)=2, \operatorname{deg}(v)=6$. The third edge partition
$E_{3}(\operatorname{DSL}(n))$ contains $54 n^{2}-66 n+24$ edges $u v$, where $\operatorname{deg}(u)=$ $3, \operatorname{deg}(v)=6$. The fourth edge partition $E_{4}(\operatorname{DSL}(n))$ contains $54 n^{2}-78 n+30$ edges $u v$, where $\operatorname{deg}(u)=\operatorname{deg}(v)=6$. Now using (2)-(4), we have

$$
H M(G)=\sum_{u v \in E(G)}[\operatorname{deg}(u)+\operatorname{deg}(v)]^{2},
$$

$H M(\operatorname{DSL}(n))$

$$
\begin{aligned}
& =\sum_{u v \in E_{1}(\operatorname{DSL}(n))}[\operatorname{deg}(u)+\operatorname{deg}(v)]^{2} \\
& +\sum_{u v \in E_{2}(\operatorname{DSL}(n))}[\operatorname{deg}(u)+\operatorname{deg}(v)]^{2} \\
& +\sum_{u v \in E_{3}(\operatorname{DSL}(n))}[\operatorname{deg}(u)+\operatorname{deg}(v)]^{2} \\
& \quad+\sum_{u v \in E_{4}(\operatorname{DSL}(n))}[\operatorname{deg}(u)+\operatorname{deg}(v)]^{2},
\end{aligned}
$$

$H M(\operatorname{DSL}(n))$

$$
\begin{aligned}
= & 5^{2}\left|E_{1}(\operatorname{DSL}(n))\right|+8^{2}\left|E_{2}(\operatorname{DSL}(n))\right| \\
& +9^{2}\left|E_{3}(\operatorname{DSL}(n))\right|+12^{2}\left|E_{4}(\operatorname{DSL}(n))\right| \\
= & 11070 n^{2}-13452 n+4746,
\end{aligned}
$$$$
P M_{1}(G)=\prod_{u v \in E(G)}[\operatorname{deg}(u)+\operatorname{deg}(v)],
$$$$
P M_{1}(\operatorname{DSL}(n))
$$$$
=\prod_{u v \in E_{1}(\operatorname{DSL}(n))}[\operatorname{deg}(u)+\operatorname{deg}(v)]
$$$$
\times \prod_{u v \in E_{2}(\operatorname{DSL}(n))}[\operatorname{deg}(u)+\operatorname{deg}(v)]
$$$$
\times \prod_{u v \in E_{3}(\operatorname{DSL}(n))}[\operatorname{deg}(u)+\operatorname{deg}(v)]
$$$$
\times \prod_{u v \in E_{4}(\operatorname{DSL}(n))}[\operatorname{deg}(u)+\operatorname{deg}(v)]
$$$$
=5^{\left|E_{1}(\operatorname{DSL}(n))\right|} \times 8^{\left|E_{2}(\operatorname{DSL}(n))\right|} \times 9^{\left|E_{3}(\operatorname{DSL}(n))\right|}
$$$$
\times 12^{\left|E_{4}(\operatorname{DSL}(n))\right|}
$$$$
=5^{(12 n-6)} \times 8^{(24 n-12)} \times 9^{\left(54 n^{2}-66 n+24\right)}
$$$$
\times 12^{\left(54 n^{2}-78 n+30\right)},
$$$$
P M_{2}(G)=\prod_{u v \in E(G)}[\operatorname{deg}(u) \times \operatorname{deg}(v)],
$$

$P M_{2}(\operatorname{DSL}(n))$

$$
=\prod_{u v \in \mathrm{E}_{1}(\operatorname{DSL}(n))}[\operatorname{deg}(u) \times \operatorname{deg}(v)]
$$




$$
\begin{aligned}
& \times \prod_{u v \in E_{2}(\operatorname{DSL}(n))}[\operatorname{deg}(u) \times \operatorname{deg}(v)] \\
& \times \prod_{u v \in E_{3}(\operatorname{DSL}(n))}[\operatorname{deg}(u) \times \operatorname{deg}(v)] \\
& \times \prod_{u v \in E_{4}(\operatorname{DSL}(n))}[\operatorname{deg}(u) \times \operatorname{deg}(v)] \\
& =6^{\left|E_{1}(\operatorname{DSL}(n))\right|} \times 12^{\left|E_{2}(\operatorname{DSL}(n))\right|} \times 18^{\left|E_{3}(\operatorname{DSL}(n))\right|} \\
& \times 36^{\left|E_{4}(\operatorname{DSL}(n))\right|} \\
& =6^{(12 n-6)} \times 12^{(24 n-12)} \times 18^{\left(54 n^{2}-66 n+24\right)} \\
& \times 36^{\left(54 n^{2}-78 n+30\right)}, \\
& M_{1}(G, x)=\sum_{u v \in E(G)} x^{[\operatorname{deg}(u)+\operatorname{deg}(v)]}, \\
& M_{1}(\operatorname{DSL}(n), x) \\
& =\sum_{u v \in E_{1}(\operatorname{DSL}(n))} x^{[\operatorname{deg}(u)+\operatorname{deg}(v)]} \\
& +\sum_{u v \in E_{2}(\operatorname{DSL}(n))} x^{[\operatorname{deg}(u)+\operatorname{deg}(v)]} \\
& +\sum_{u v \in E_{3}(\operatorname{DSL}(n))} x^{[\operatorname{deg}(u)+\operatorname{deg}(v)]} \\
& +\sum_{u v \in E_{4}(\operatorname{DSL}(n))} x^{[\operatorname{deg}(u)+\operatorname{deg}(v)]} \\
& =\sum_{u v \in E_{1}(\operatorname{DSL}(n))} x^{5}+\sum_{u v \in E_{2}(\operatorname{DSL}(n))} x^{8}+\sum_{u v \in E_{3}(\operatorname{DSL}(n))} x^{9} \\
& +\sum_{u v \in E_{4}(\operatorname{DSL}(n))} x^{12} \\
& =\left|E_{1}(\operatorname{DSL}(n))\right| x^{5}+\left|E_{2}(\operatorname{DSL}(n))\right| x^{8} \\
& +\left|E_{3}(\operatorname{DSL}(n))\right| x^{9}+\left|E_{4}(\operatorname{DSL}(n))\right| x^{12} \\
& =(12 n-6) x^{5}+(24 n-12) x^{8} \\
& +\left(54 n^{2}-66 n+24\right) x^{9} \\
& +\left(54 n^{2}-78 n+30\right) x^{12}, \\
& M_{2}(G, x)=\sum_{u v \in E(G)} x^{[\operatorname{deg}(u) \times \operatorname{deg}(v)]}, \\
& M_{2}(\operatorname{DSL}(n), x) \\
& =\sum_{u v \in E_{1}(\operatorname{DSL}(n))} x^{[\operatorname{deg}(u) \times \operatorname{deg}(v)]} \\
& +\sum_{u v \in E_{2}(\operatorname{DSL}(n))} x^{[\operatorname{deg}(u) \times \operatorname{deg}(v)]} \\
& +\sum_{u v \in E_{3}(\operatorname{DSL}(n))} x^{[\operatorname{deg}(u) \times \operatorname{deg}(v)]}
\end{aligned}
$$

$$
\begin{aligned}
& +\sum_{u v \in E_{4}(\operatorname{DSL}(n))} x^{[\operatorname{deg}(u) \times \operatorname{deg}(v)]} \\
= & \sum_{u v \in E_{1}(\operatorname{DSL}(n))} x^{6}+\sum_{u v \in E_{2}(\operatorname{DSL}(n))} x^{12}+\sum_{u v \in E_{3}(\operatorname{DSL}(n))} x^{18} \\
& +\sum_{u v \in E_{4}(\operatorname{DSL}(n))} x^{36} \\
= & \left|E_{1}(\operatorname{DSL}(n))\right| x^{6}+\left|E_{2}(\operatorname{DSL}(n))\right| x^{12} \\
& +\left|E_{3}(\operatorname{DSL}(n))\right| x^{18}+\left|E_{4}(\operatorname{DSL}(n))\right| x^{36} \\
= & (12 n-6) x^{6}+(24 n-12) x^{12} \\
& +\left(54 n^{2}-66 n+24\right) x^{18} \\
& +\left(54 n^{2}-78 n+30\right) x^{36} .
\end{aligned}
$$

\section{Regular Triangulene Oxide Network $\operatorname{RTOX}(n)$}

In 2015, Baig et al. [34] computed general Randić index, atom-bond connectivity $(\mathrm{ABC})$ index, and geometric arithmetic (GA) index of regular triangulene oxide network $\operatorname{RTOX}(n)$. Now we compute hyper Zagreb index $H M(G)$, first multiple Zagreb index $P M_{1}(G)$, second multiple Zagreb index $P M_{2}(G)$, and Zagreb polynomials $M_{1}(G, x)$ and $M_{2}(G, x)$ for regular triangulene oxide network $\operatorname{RTOX}(n)$.

Theorem 5. Consider the regular triangulene oxide network $\operatorname{RTOX}(n)$ for $n \geq 2$. Then

$$
\begin{gathered}
H M(R T O X(n))=192 n^{2}+216 n-96, \\
P M_{1}(\operatorname{RTOX}(n))=4^{2} \times 6^{6 n} \times 8^{3 n^{2}-2}, \\
P M_{2}(\operatorname{RTOX}(n))=4^{2} \times 8^{6 n} \times 16^{3 n^{2}-2}, \\
M_{1}(\operatorname{RTOX}(n), x)=2 x^{4}+6 n x^{6}+\left(3 n^{2}-2\right) x^{8}, \\
M_{2}(\operatorname{RTOX}(n), x)=2 x^{4}+6 n x^{8}+\left(3 n^{2}-2\right) x^{16} .
\end{gathered}
$$

Proof. Let RTOX $(n)$ be the regular triangulene oxide network; see Figure 4 . The number of vertices and edges in $\operatorname{RTOX}(n)$ is $\left(3 n^{2}+9 n+2\right) / 2$ and $3 n^{2}+6 n$, respectively. There are three types of edges in $E(\operatorname{RTOX}(n))$ based on degrees of end vertices of each edge; that is, $E(\operatorname{RTOX}(n))=$ $E_{1}(\operatorname{RTOX}(n)) \cup E_{2}(\operatorname{RTOX}(n)) \cup E_{3}(\operatorname{RTOX}(n))$. The edge partition $E_{1}(\operatorname{RTOX}(n))$ contains 2 edges $u v$, where $\operatorname{deg}(u)=$ $\operatorname{deg}(v)=2$; the edge partition $E_{2}(\operatorname{RTOX}(n))$ contains $6 n$ edges $u v$, where $\operatorname{deg}(u)=2$ and $\operatorname{deg}(v)=4$, and the edge 


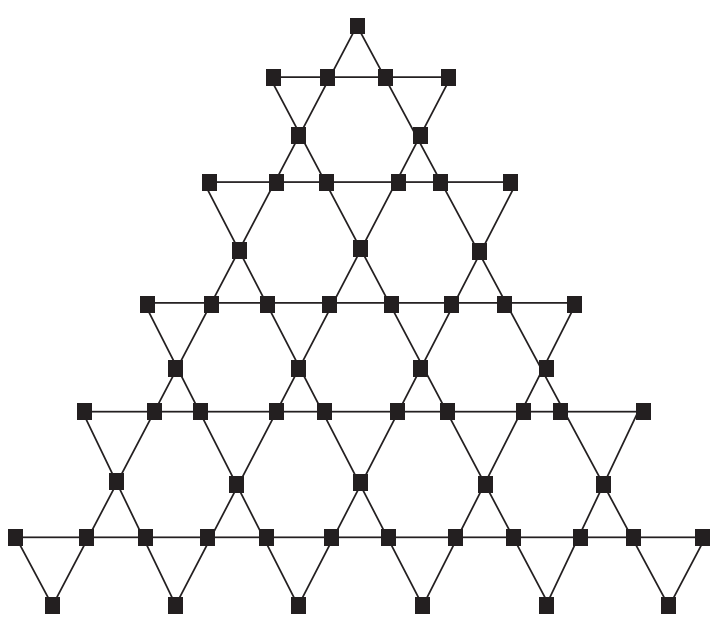

Figure 4: Regular triangulene oxide network (RTOX(5)).

partition $E_{3}(\operatorname{RTOX}(n))$ contains $3 n^{2}-2$ edges $u v$, where $\operatorname{deg}(u)=\operatorname{deg}(v)=4$.

Now using (2)-(4), we have

$$
\begin{aligned}
& H M(G)=\sum_{u v \in E(G)}[\operatorname{deg}(u)+\operatorname{deg}(v)]^{2}, \\
& H M(\operatorname{RTOX}(n)) \\
& =\sum_{u v \in E_{1}(\operatorname{RTOX}(n))}[\operatorname{deg}(u)+\operatorname{deg}(v)]^{2} \\
& \quad+\sum_{u v \in E_{2}(\operatorname{RTOX}(n))}[\operatorname{deg}(u)+\operatorname{deg}(v)]^{2} \\
& \quad+\sum_{u v \in E_{3}(\operatorname{RTOX}(n))}[\operatorname{deg}(u)+\operatorname{deg}(v)]^{2} \\
& =4^{2}\left|E_{1}(\operatorname{RTOX}(n))\right|+6^{2}\left|E_{2}(\operatorname{RTOX}(n))\right| \\
& +8^{2}\left|E_{3}(\operatorname{RTOX}(n))\right| \\
& =16(2)+36(6 n)+64\left(3 n^{2}-2\right) \\
& =192 n^{2}+216 n-96,
\end{aligned}
$$$$
P M_{1}(G)=\prod_{u v \in E(G)}[\operatorname{deg}(u)+\operatorname{deg}(v)]
$$$$
P M_{1}(\mathrm{RTOX}(n))
$$$$
=\prod_{u v \in E_{1}(\operatorname{RTOX}(n))}[\operatorname{deg}(u)+\operatorname{deg}(v)]
$$$$
\times \prod_{u v \in E_{2}(\operatorname{RTOX}(n))}[\operatorname{deg}(u)+\operatorname{deg}(v)]
$$$$
\times \prod_{u v \in E_{3}(\operatorname{RTOX}(n))}[\operatorname{deg}(u)+\operatorname{deg}(v)]
$$

$$
\begin{aligned}
= & 4^{\left|E_{1}(\operatorname{RTOX}(n))\right|} \times 6^{\left|E_{2}(\operatorname{RTOX}(n))\right|} \times 8^{\left|E_{3}(\operatorname{RTOX}(n))\right|} \\
= & 4^{2} \times 6^{6 n} \times 8^{3 n^{2}-2}, \\
P M_{2}(G)=\prod_{u v \in E(G)}[\operatorname{deg}(u) \times \operatorname{deg}(v)] & \\
P M_{2} & (\operatorname{RTOX}(n)) \\
= & \prod_{u v \in E_{1}(\operatorname{RTOX}(n))}[\operatorname{deg}(u) \times \operatorname{deg}(v)] \\
& \times \prod_{u v \in E_{2}(\operatorname{RTOX}(n))}[\operatorname{deg}(u) \times \operatorname{deg}(v)] \\
& \times \prod_{u v \in E_{3}(\operatorname{RTOX}(n))}[\operatorname{deg}(u) \times \operatorname{deg}(v)] \\
= & 4^{\left|E_{1}(\operatorname{RTOX}(n))\right|} \times 8^{\left|E_{2}(\operatorname{RTOX}(n))\right|} \times 16^{\left|E_{3}(\operatorname{RTOX}(n))\right|} \\
= & 4^{2} \times 8^{6 n} \times 16^{3 n^{2}-2},
\end{aligned}
$$$$
M_{1}(G, x)=\sum_{u v \in E(G)} x^{[\operatorname{deg}(u)+\operatorname{deg}(v)]},
$$$$
M_{1}(\operatorname{RTOX}(n), x)
$$$$
=\sum_{u v \in E_{1}(\operatorname{RTOX}(n))} x^{[\operatorname{deg}(u)+\operatorname{deg}(v)]}
$$$$
+\sum_{u v \in E_{2}(\operatorname{RTOX}(n))} x^{[\operatorname{deg}(u)+\operatorname{deg}(v)]}
$$$$
+\sum_{u v \in E_{3}(\operatorname{RTOX}(n))} x^{[\operatorname{deg}(u)+\operatorname{deg}(v)]}
$$$$
=\sum_{u v \in E_{1}(\operatorname{RTOX}(n))} x^{4}+\sum_{u v \in E_{2}(\operatorname{RTOX}(n))} x^{6}
$$$$
+\sum_{u v \in E_{3}(\operatorname{RTOX}(n))} x^{8}
$$$$
=\left|E_{1}(\operatorname{RTOX}(n))\right| x^{4}+\left|E_{2}(\operatorname{RTOX}(n))\right| x^{6}
$$$$
+\left|E_{3}(\operatorname{RTOX}(n))\right| x^{8}
$$$$
=2 x^{4}+6 n x^{6}+\left(3 n^{2}-2\right) x^{8} \text {, }
$$$$
M_{2}(G, x)=\sum_{u v \in E(G)} x^{[\operatorname{deg}(u) \times \operatorname{deg}(v)]},
$$$$
M_{2}(\operatorname{RTOX}(n), x)
$$$$
=\sum_{u v \in E_{1}(\operatorname{RTOX}(n))} x^{[\operatorname{deg}(u) \times \operatorname{deg}(v)]}
$$$$
+\sum_{u v \in E_{2}(\operatorname{RTOX}(n))} x^{[\operatorname{deg}(u) \times \operatorname{deg}(v)]}
$$$$
+\sum_{u v \in E_{3}(\operatorname{RTOX}(n))} x^{[\operatorname{deg}(u) \times \operatorname{deg}(v)]}
$$$$
=\sum_{u v \in E_{1}(\operatorname{RTOX}(n))} x^{4}+\sum_{u v \in E_{2}(\operatorname{RTOX}(n))} x^{8}
$$ 


$$
\begin{aligned}
& +\sum_{u v \in E_{3}(\operatorname{RTOX}(n))} x^{16} \\
= & \left|E_{1}(\operatorname{RTOX}(n))\right| x^{4}+\left|E_{2}(\operatorname{RTOX}(n))\right| x^{8} \\
& +\left|E_{2}(\operatorname{RTOX}(n))\right| x^{16} \\
= & 2 x^{4}+6 n x^{8}+\left(3 n^{2}-2\right) x^{16} .
\end{aligned}
$$

\section{Conclusion}

In this paper we determined hyper Zagreb index $H M(G)$, first multiple Zagreb index $P M_{1}(G)$, second multiple Zagreb index $P M_{2}(G)$, and Zagreb polynomials $M_{1}(G, x)$ and $M_{2}(G, x)$ for nanotubes $\mathbb{N A}_{m}^{n}$ and $\mathbb{N C}_{m}^{n}$, dominating oxide network $\operatorname{DOX}(n)$, dominating silicate network $\operatorname{DSL}(n)$, and regular triangulene oxide network $\operatorname{RTOX}(n)$.

In the future, we are interested in designing some new architectures/networks and then studying their topological indices which will be quite helpful to understand their underlying topologies.

\section{Competing Interests}

The authors declare that they have no competing interests.

\section{References}

[1] M.-S. Chen and K. G. Shin, "Adaptive fault-tolerant routing in hypercube multicomputers," Institute of Electrical and Electronics Engineers. Transactions on Computers, vol. 39, no. 12, pp. 1406-1416, 1990.

[2] M. Ghorbani and N. Azimi, "Note on multiple zagreb indices," Iranian Journal of Mathematical Chemistry, vol. 3, no. 2, pp. 137143, 2012.

[3] K. C. Das and I. Gutman, "Some properties of the second Zagreb index," MATCH. Communications in Mathematical and in Computer Chemistry, no. 52, pp. 103-112, 2004.

[4] I. Gutman and N. Trinajstić, "Graph theory and molecular orbitals. Total $\varphi$-electron energy of alternant hydrocarbons," Chemical Physics Letters, vol. 17, no. 4, pp. 535-538, 1972.

[5] I. Gutman, J. Tošović, S. Radenković, and S. Marković, "On atombond connectivity index and its chemical applicability," Indian Journal of Chemistry, vol. 51, pp. 690-694, 2012.

[6] H. Wiener, "Structural determination of paraffin boiling points," Journal of the American Chemical Society, vol. 69, no. 1, pp. 1720, 1947.

[7] A. A. Dobrynin, R. Entringer, and I. Gutman, "Wiener index of trees: theory and applications," Acta Applicandae Mathematicae, vol. 66 , no. 3, pp. 211-249, 2001.

[8] I. Gutman and O. E. Polansky, Mathematical Concepts in Organic Chemistry, Springer, Berlin, Germany, 1986.

[9] G. H. Shirdel, H. R. Pour, and A. M. Sayadi, "The hyper-zagreb index of graph operations," Iranian Journal of Mathematical Chemistry, vol. 4, no. 2, pp. 213-220, 2013.

[10] M. Eliasi, A. Iranmanesh, and I. Gutman, "Multiplicative versions of first Zagreb index," MATCH. Communications in Mathematical and in Computer Chemistry, vol. 68, no. 1, pp. 217230, 2012.
[11] T. Al-Fozan, P. Manuel, I. Rajasingh, and R. S. Rajan, "A new technique to compute padmakar-ivan index and szeged index of pericondensed benzenoid graphs," Journal of Computational and Theoretical Nanoscience, vol. 11, no. 2, pp. 533-539, 2014.

[12] A. R. Ashrafi and P. Nikzad, "Connectivity index of the family of dendrimer nanostars," Digest Journal of Nanomaterials and Biostructures, vol. 4, no. 2, pp. 269-273, 2009.

[13] I. Gutman, "Degree-based topological indices," Croatica Chemica Acta, vol. 86, no. 4, pp. 351-361, 2013.

[14] S. Hayat and M. Imran, "Computation of topological indices of certain networks," Applied Mathematics and Computation, vol. 240, pp. 213-228, 2014.

[15] M. Aouchiche and P. Hansen, "On a conjecture about the Randić index," Discrete Mathematics, vol. 307, no. 2, pp. 262-265, 2007.

[16] M. Aouchiche, P. Hansen, and M. Zheng, "Variable neighborhood search for extremal graphs," MATCH Communications in Mathematical and in Computer Chemistry, vol. 58, pp. 83-102, 2007.

[17] M. Bača, J. Horváthová, M. Mokrišová, and A. z. Suhányiová, "On topological indices of fullerenes," Applied Mathematics and Computation, vol. 251, pp. 154-161, 2015.

[18] M. Bača, J. Horváthová, M. Mokrišová, A. SemanicovFenovckov, and A. Suhányiová, "On topological indices of carbon nanotube network," Canadian Journal of Chemistry, vol. 93, pp. 1-4, 2015.

[19] B. Bollobás and P. Erdös, "Graphs of extremal weights," Ars Combinatoria, vol. 50, pp. 225-233, 1998.

[20] A. Graovac, M. Ghorbani, and M. A. Hosseinzadeh, "Computing fifth geometric-arithmetic index for nanostar dendrimers," Journal of Mathematical NanoScience, vol. 1, no. 1, pp. 33-42, 2011.

[21] S. Hayat and M. Imran, "Computation of certain topological indices of nanotubes covered by $\mathrm{C}_{5}$ and $\mathrm{C}_{7}$," Journal of Computational and Theoretical Nanoscience, vol. 12, no. 4, pp. 533-541, 2015.

[22] M. Imran, S. Hayat, and M. Y. H. Mailk, "On topological indices of certain interconnection networks," Applied Mathematics and Computation, vol. 244, pp. 936-951, 2014.

[23] J. Rada, R. Cruz, and I. Gutman, "Benzenoid systems with extremal vertex-degree-based topological indices," MATCH. Communications in Mathematical and in Computer Chemistry, vol. 72, no. 1, pp. 125-136, 2014.

[24] J. Rada and R. Cruz, "Vertex-degree-based topological indices over graphs," MATCH. Communications in Mathematical and in Computer Chemistry, vol. 72, no. 3, pp. 603-616, 2014.

[25] M. Randić, "On characterization of molecular branching," Journal of the American Chemical Society, vol. 97, no. 23, pp. 6609-6615, 1975.

[26] M. K. Siddiqui, M. Imran, and A. Ahmad, "On ZAGreb indices, ZAGreb polynomials of some nanostar dendrimers," Applied Mathematics and Computation, vol. 280, pp. 132-139, 2016.

[27] B. Wu and L. Zhang, "Unicyclic graphs with minimum general Randić index," MATCH. Communications in Mathematical and in Computer Chemistry, vol. 54, no. 2, pp. 455-464, 2005.

[28] M. Deza, P. W. Fowler, A. Rassat, and K. M. Rogers, "Fullerenes as tilings of surfaces," Journal of Chemical Information and Computer Sciences, vol. 40, no. 3, pp. 550-558, 2000.

[29] W. Gao, Y. Guo, and K. Wang, "Ontology algorithm using singular value decomposition and applied in multidisciplinary," Cluster Computing, vol. 19, no. 4, pp. 2201-2210, 2016. 
[30] W. Gao, A. Q. Baig, H. Ali, W. Sajjad, and M. R. Farahani, "Margin based ontology sparse vector learning algorithm and applied in biology science," Saudi Journal of Biological Sciences, vol. 24, no. 1, pp. 132-138, 2017.

[31] W. Gao and W. Wang, "The eccentric connectivity polynomial of two classes of nanotubes," Chaos, Solitons \&; Fractals, vol. 89, pp. 290-294, 2016.

[32] W. Gao and W. F. Wang, "The fifth geometric-arithmetic index of bridge graph and carbon nanocones," Journal of Difference Equations and Applications, pp. 1-10, 2016.

[33] D. J. Klein, "Elemental benzenoids," Journal of Chemical Information and Modeling, vol. 34, no. 2, pp. 453-459, 1994.

[34] A. Q. Baig, M. Imran, and H. Ali, "On topological indices of poly oxide, poly silicate, DOX, and DSL networks," Canadian Journal of Chemistry, vol. 93, no. 7, pp. 730-739, 2015. 

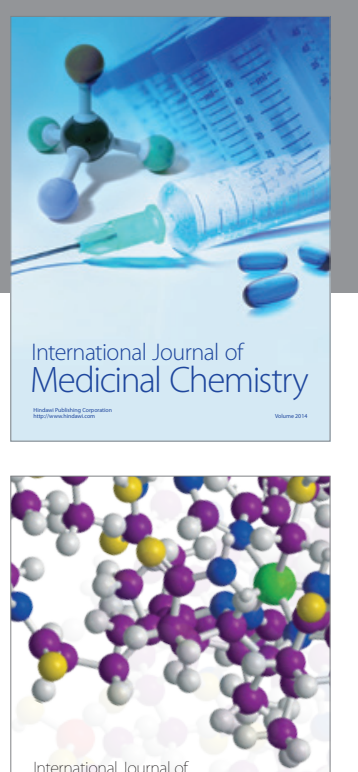

Carbohydrate Chemistry

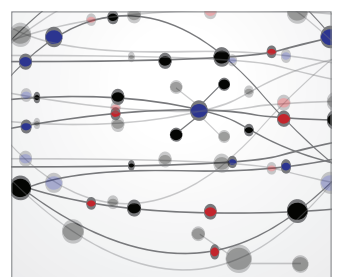

The Scientific World Journal
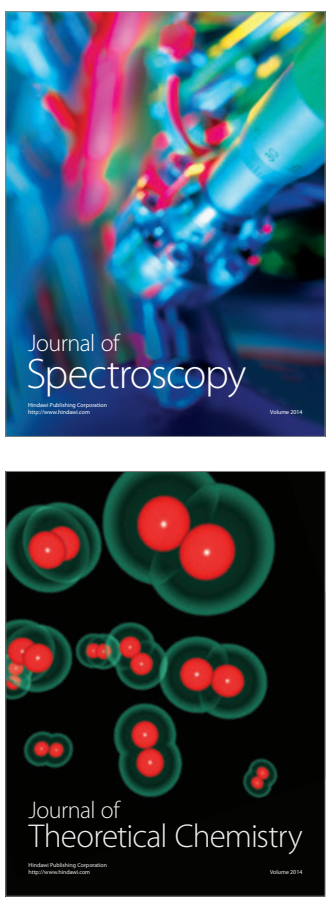
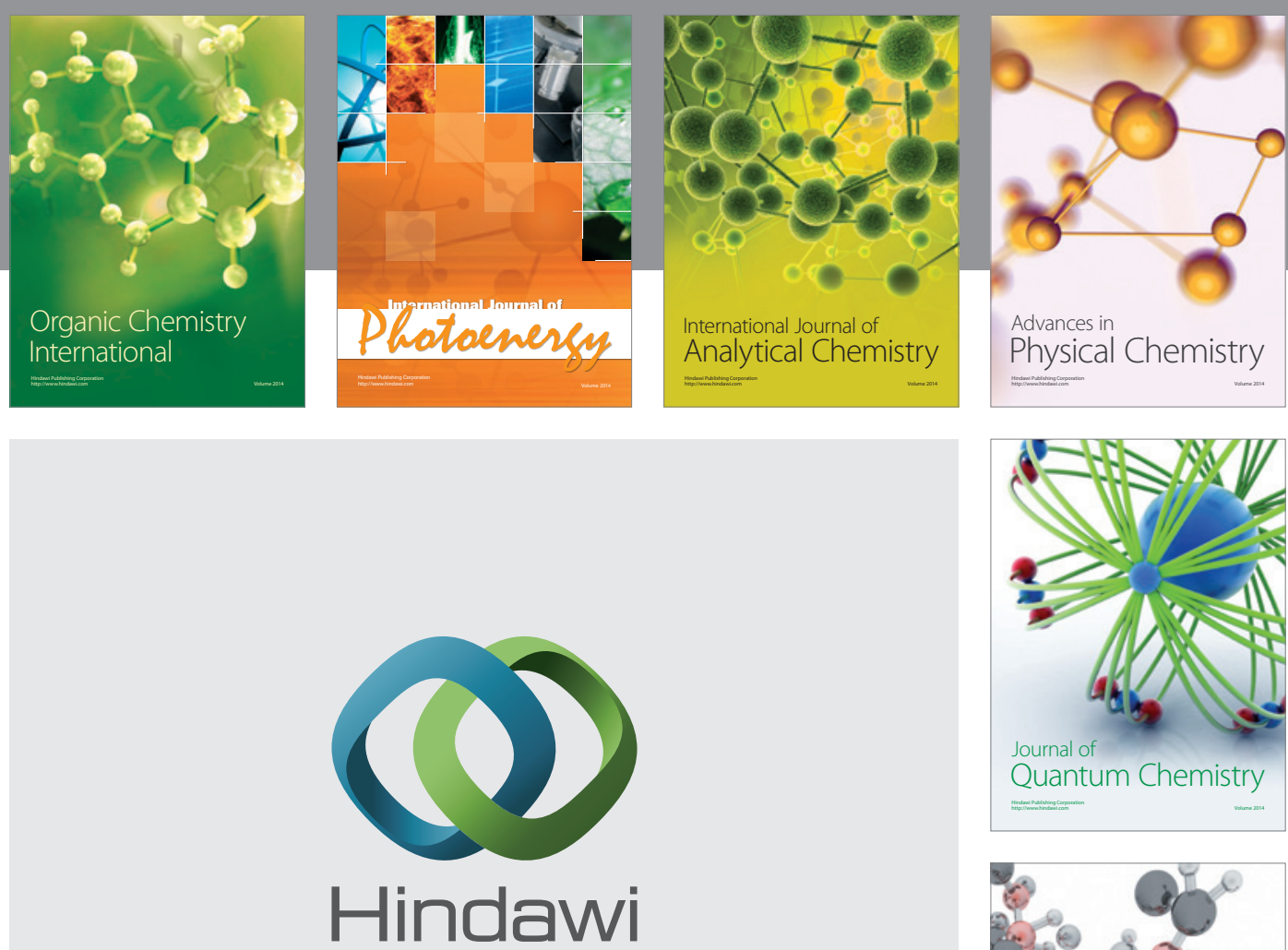

Submit your manuscripts at

https://www.hindawi.com

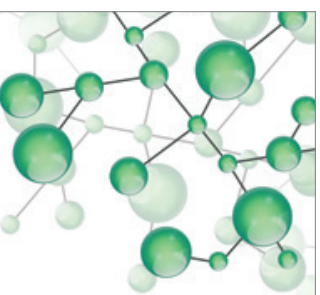

International Journal of

Inorganic Chemistry
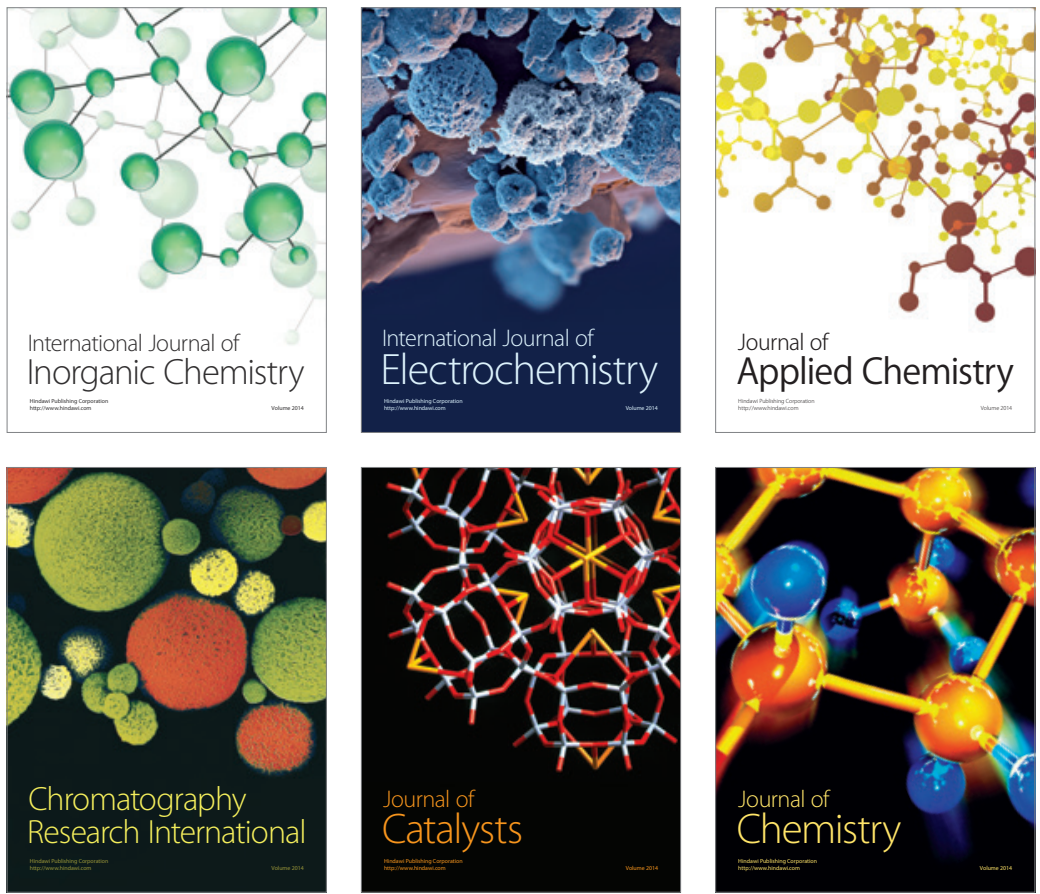

Journal of

Applied Chemistry
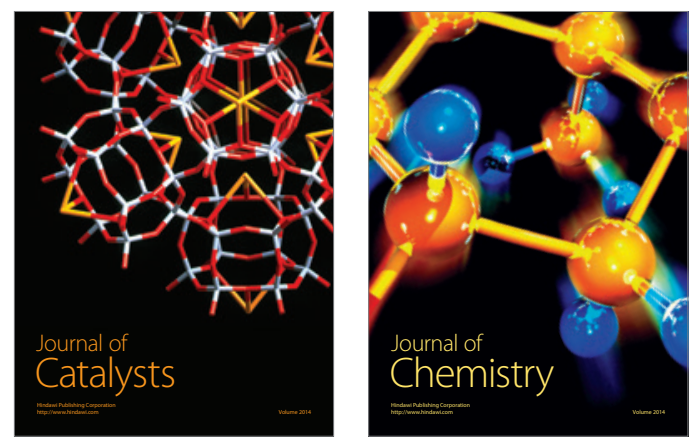
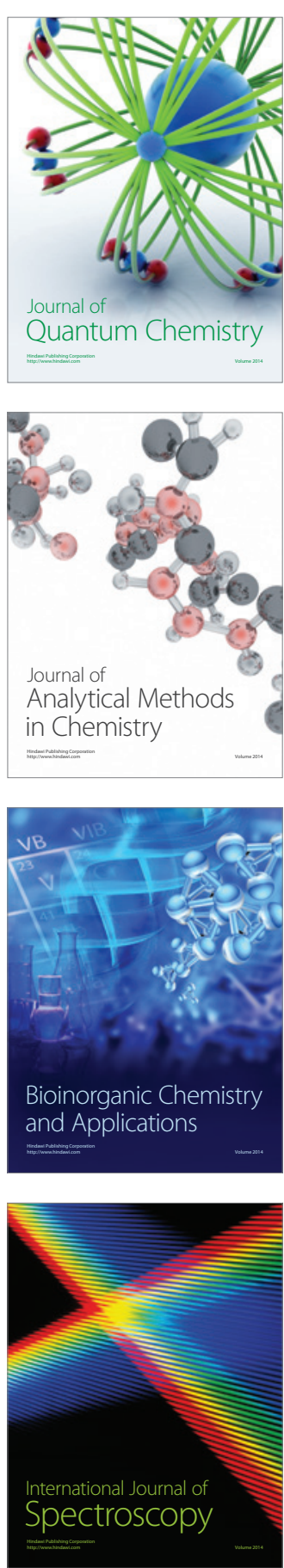